\title{
Analysis of graph parameters associated with zero-divisor graphs of commutative rings
}

\author{
Sumeyye Aykac ${ }^{1}$ and Nihat Akgunes ${ }^{2}$ \\ ${ }^{1}$ Selcuk University, Faculty of Science, Department of Mathematics, Konya, Turkey \\ ${ }^{2}$ Necmettin Erbakan University, Faculty of Science, Department of Mathematics-Computer Sciences, Konya, Turkey
}

Received: 21 March 2018, Accepted: 3 April 2018

Published online: 16 April 2018.

\begin{abstract}
In this paper, the diameter, radius, girth, maximum and minimum degrees, domination number, chromatic number, clique number, degree sequence, irregularity index of $\Gamma\left(\mathbb{Z}_{p^{2}} \times \mathbb{Z}_{q^{2}}\right)$ have been established, where $p$ and $q$ are prime.
\end{abstract}

Keywords: Zero-divisor graph, ring, girth, graph.

\section{Introduction}

Beck established the notion of the zero divisor graph $\Gamma(R)$ of a commutative ring $R$, in [5]. Then, zero-divisor graphs over commutative and non-commutative rings have been examined widely in terms of spectral and non-spectral graph properties. Let $R$ be a commutative ring with identity, and let $Z(R)$ be the set of its zero-divisors. In this case the zerodivisor graph $\Gamma(R)$ is actually an (undirected) graph with vertices $x, y \in Z(R)^{*}=Z(R) \backslash\{0\}$ such that $x$ and $y$ are adjacent if and only if $x y=0$. It is clear to see that $\Gamma(R)$ is the empty graph if and only if $R$ is an integral domain. After that, by [2], a nonempty $\Gamma(R)$ is finite if and only if $R$ is finite and not a field. Additively, in [2], Anderson et al. proved that if $R$ is commutative then $\Gamma(R)$ is always connected. Afterwards, Anderson and Badawi [3] examined $\Gamma(R)$ for rings $R$ with nonzero zero-divisors which satisfy certain divisibility provisions between elements of $R$ or comparability provisions between ideals or prime ideals of $R$. Lately, Sharma et al. [10] have been examined the adjacency matrix of a zero-divisor graph on finite commutative rings. In fact, they have been tried to find a detailed description for the adjacency matrix of zero-divisor graphs obtained from the ring $\mathbb{Z}_{p} \times \mathbb{Z}_{p}$, where $p$ is a prime. The zero-divisor graph of a commutative ring has been examined comprehensively by several authors, e.g. [11,1,6,7,9]. In [8], Akgunes et al. presented energy, domination number, diameter, Randić index, girth, maximum degree, Estrada index and Randić energy for zero-divisor graphs obtained from the ring $\mathbb{Z}_{p} \times \mathbb{Z}_{q}$, where $p$ and $q$ are distinct primes. In this paper, degree sequence, irregularity index, cromatic number, diameter, girth, radius, maximum and minimum degrees, domination number, clique number, we will mainly prove some results over the zero-divisor graphs $\Gamma\left(\mathbb{Z}_{p^{2}} \times \mathbb{Z}_{q^{2}}\right)$ for distinct primes $p$ and $q$.

\section{Some spectral properties of $\Gamma\left(\mathbb{Z}_{p^{2}} \times \mathbb{Z}_{q^{2}}\right)$}

In this section, by considering the graph $\Gamma\left(\mathbb{Z}_{p^{2}} \times \mathbb{Z}_{q^{2}}\right)$, where $p$ and $q$ is prime, we will mainly deal with the graph properties, namely diameter, girth, radius, maximum and minimum degrees, domination number, click number, irrequality index, degree sequence and finally chromatic number. It is quite well known that, for any graph $\Gamma$, most of these proporties 
can be obtained by checking the distance or the total number of the vertices. So, the methods in the proofs of the results in this section will be followed by this idea.

Definition 1. When $p$ and $q$ are prime, the adjacent of the graph $\Gamma\left(\mathbb{Z}_{p^{2}} \times \mathbb{Z}_{q^{2}}\right)$ of the ring $\mathbb{Z}_{p^{2}} \times \mathbb{Z}_{q^{2}}$ are as follows.

(i) $(0, j) \sim(i, 0)$ where $0<j<q^{2}$ and $0<i<p^{2}$.

(ii) $\left(k_{1} p, j\right) \sim\left(k_{2} p, 0\right)$ where $0 \leq j<q^{2}$ and $0<k_{1}, k_{2}<p$.

(iii) $\left(0, t_{1} q\right) \sim\left(i, t_{2} q\right)$ where $0 \leq i<p^{2}$ and $0<t_{1}, t_{2}<q$.

(iv) $\left(k_{1} p, t_{1} q\right) \sim\left(k_{2} p, t_{2} q\right)$ where $0 \leq k_{1}, k_{2}<p$ and $0<t_{1}, t_{2}<q$.

By definition, it is clear that proof of the following theorem.

Theorem 1. The distance between any two vertices of the $\Gamma\left(\mathbb{Z}_{p^{2}} \times \mathbb{Z}_{q^{2}}\right)$ are as follows

(i) $d\left(\left(0, j_{1}\right),\left(0, j_{2}\right)\right)=2$ where $0<j_{1}, j_{2}<q^{2}, j_{1}, j_{2} \neq t q(0<t<q)$.

(ii) $d((0, j),(i, t q))=3$ where $0<j<q^{2}, 0<i<p^{2}, 0<t<q$ and $j \neq t q$.

(iii) $d((0, j),(k p, j))=2$ where $0<j<q^{2}, 0<k<p, 0<t<q$ and $j \neq t q$.

(iv) $d((0, j),(0, t q))=2$ where $0<j<q^{2}, 0<t<q$ and $j \neq t q$.

(v) $d\left(\left(i_{1}, 0\right),\left(i_{2}, 0\right)\right)=2$ where $0<i_{1}, i_{2}<p^{2}, i_{1}, i_{2} \neq k p(0<k<p)$.

(vi) $d((i, 0),(k p, j))=3$ where $0<i<p^{2}, 0<k<p, 0<j<q^{2}, i \neq k p$.

(vii) $d((i, 0),(k p, t q))=2$ where $0<i<p^{2}, 0<k<p, 0 \leq t<q, i \neq k p$.

(viii) $d\left(\left(i_{1}, 0\right),\left(i_{2}, t q\right)\right)=2$ where $0<i_{1}, i_{2}<p^{2}, 0<t<q, i_{1}, i_{2} \neq k p(0<k<p)$.

(ix) $d((k p, j),(0, t q))=2$ where $0<k<p, 0<j<q^{2}, 0<t<q, j \neq t q$.

(x) $d((k p, j),(i, t q))=3$ where $0<i<p^{2}, 0<k<p, 0<j<q^{2}, 0<t<q, i \neq k p$.

(xi) $d((k p, j),(k p, j))=2$ where $0<j<q^{2}, 0<k<p, 0<t<q, j \neq t q$.

(xii) $d((i, t q),(i, t q))=2$ where $0<i<p^{2}, 0<k<p, 0<t<q, i \neq k p$.

We first recall that, for any simple graph $\Gamma$, the diameter(length of the shortest path) between two vertices $u_{1}, u_{2}$ of $\Gamma$ is defined by

$$
\operatorname{diam}(\Gamma)=\max \left\{d_{\Gamma}\left(u_{1}, u_{2}\right): u_{1} \text { and } u_{2} \text { are vertices of } G\right\}
$$

We thus obtain the following result.

Theorem 2. For any ring $\mathbb{Z}_{p^{2}} \times \mathbb{Z}_{q^{2}}$, the diameter of the graph $\Gamma\left(\mathbb{Z}_{p^{2}} \times \mathbb{Z}_{q^{2}}\right)$ is 3 .

Proof. We consider vertex of $\Gamma\left(\mathbb{Z}_{p^{2}} \times \mathbb{Z}_{q^{2}}\right)$. Let $(i, t q)$ and $(k p, j)$ for $i \neq k p, j \neq t q$ be any two vertices in $\Gamma\left(\mathbb{Z}_{p^{2}} \times \mathbb{Z}_{q^{2}}\right)$. For $0<k<p, 0<t<q, 0<i<p^{2}, 0<j<q^{2}$; every $(k p, j) \sim(k p, 0)$ and so $d_{G}((k p, j),(k p, 0))=1$, every $(i, t q) \sim(0, t q)$ and so $d_{G}((i, t q),(0, t q))=1$, every $(0, t q) \sim(k p, 0)$ and so $d_{G}((0, t q),(k p, 0))=1$. Because $(k p, j) .(k p, 0)=(0,0)$, $(i, t q) \cdot(0, t q)=(0,0)$ and $(0, t q) \cdot(k p, 0)=(0,0)$. We finally get $\operatorname{diam}\left(\Gamma\left(\mathbb{Z}_{p^{2}} \times \mathbb{Z}_{q^{2}}\right)\right)=3$, as required.

The eccentricity is the maximum distance between $u_{1}$ and any other vertex $u_{2}$ of $\Gamma$. The eccentricity of a vertex $u_{1}$ in a connected graph $\Gamma$, denoted by $\varepsilon\left(u_{1}\right)$, is the maximum distance between $u_{1}$ and any other vertex $u_{2}$ of $\Gamma$. All vertices are defined to have infinite eccentricity when $\Gamma$ is a disconnected graph. It is well known that the diameter is the maximum distance between any two vertices of $\Gamma$. It is quite clear that $\operatorname{diam}(\Gamma)$ is equal to the maximum eccentricity among all vertices of $\Gamma$. On the other hand, the minimum eccentricity is called the radius of $\Gamma$ and denoted by

$$
\operatorname{rad}(\Gamma)=\min _{u_{1}}\left\{\max _{u_{2}}\left\{d_{\Gamma}\left(u_{1}, u_{2}\right)\right\}\right\}
$$

Due to [11], for a connected graph $\Gamma$, the inequality

$$
\operatorname{rad}(\Gamma) \leq \operatorname{diam}(\Gamma)<2 \operatorname{rad}(\Gamma)
$$


always holds.

Theorem 3. The radius of the $\Gamma\left(\mathbb{Z}_{p^{2}} \times \mathbb{Z}_{q^{2}}\right)$ is 2 .

Proof. Now let's examine the eccentricity of all the vertices. $\varepsilon(k p, 0)=2$ where $0<k<p . \varepsilon(i, 0)=2$ where $i \neq k p, 0<$ $i<p^{2} . \varepsilon(0, j)=2$ where $j \neq t q, 0<j<q^{2} . \varepsilon(0, t q)=2$ where $0<t<q . \varepsilon(k p, j)=2$ where $0<k<p, j \neq t q, 0<j<q^{2}$. $\varepsilon(i, t q)=2$ where $0<t<q, i \neq k p, 0<i<p^{2} . \varepsilon(k p, t q)=2$ where $0<k<p, 0<t<q$. Minimum eccentricity is 2 . Since radius is the minimum eccentricity, $\operatorname{rad}\left(\Gamma\left(\mathbb{Z}_{p^{2}} \times \mathbb{Z}_{q^{2}}\right)\right)=2$.

Recall that the girth of a simple graph $\Gamma$ is the length of the shortest cycle contained in $\Gamma$, denoted by girth $(\Gamma)$. However, if $\Gamma$ does not contain any cycle, then the girth of it is assumed to be infinity.

Theorem 4. For any ring $\mathbb{Z}_{p^{2}} \times \mathbb{Z}_{q^{2}}$, the girth of the graph $\Gamma\left(\mathbb{Z}_{p^{2}} \times \mathbb{Z}_{q^{2}}\right)$ is 3 .

Proof. By the definition of $\Gamma\left(\mathbb{Z}_{p^{2}} \times \mathbb{Z}_{q^{2}}\right)$, since $\left(0, t_{1} q\right) \cdot\left(0, t_{2} q\right)=(0,0),\left(0, t_{2} q\right) \cdot\left(i, t_{1} q\right)=(0,0)$ and $\left(i, t_{1} q\right) \cdot\left(0, t_{1} q\right)=(0,0)$ for $0<t_{1}, t_{2}<q, 0<i<p^{2}$. We then have $\left(0, t_{1} q\right) \sim\left(0, t_{2} q\right) \sim\left(i, t_{1} q\right) \sim\left(0, t_{1} q\right)$, which implies the result, as desired.

The degree of a vertex $u_{1}$ of $\Gamma$ is the number of vertices adjacent to $u_{1}$, denoted by $\operatorname{deg}_{\Gamma}\left(u_{1}\right)$.

Theorem 5. The degree of the vertex of $\Gamma\left(\mathbb{Z}_{p^{2}} \times \mathbb{Z}_{q^{2}}\right)$ are as follows.

(i) $\operatorname{deg}(i, 0)=q^{2}-1$ where $0<i<p^{2}, i \neq k p$.

(ii) $\operatorname{deg}(k p, 0)=p q^{2}-2$ where $0<k<p$.

(iii) $\operatorname{deg}(0, j)=p^{2}-1$ where $0<j<q^{2}, j \neq t q$.

(iv) $\operatorname{deg}(0, t q)=q p^{2}-2$ where $0<t<q$.

(v) $\operatorname{deg}(k p, j)=p-1$ where $0<j<q^{2}$ and $0<k<p, j \neq t q$.

(vi) $\operatorname{deg}(i, t q)=q-1$ where $0<i<p^{2}, i \neq k p$.

(vii) $\operatorname{deg}(k p, t q)=p q-2$ where $0<t<q$ and $0<k<p$.

Proof. (i) For $i \neq k p$ and $0<i<p^{2}$, we view $(i, 0)$ vertex. $(i, 0)$ vertex adjacent only $(0, j)$. The number of $(0, j)$ vertex is $q^{2}-1$, where $0<j<q^{2}$.

(ii) For $0<k<p$, we view $(k p, 0)$ vertex. $(k p, 0)$ vertex adjacent $(0, j),(k p, 0),(k p, j)$ where $0<j<q^{2}, 0<k<p$. The number of these vertices is $\left(q^{2}-1\right)+\left(q^{2}-1\right) \cdot(p-1)+(p-2)$, which equals $p q^{2}-2$.

(iii) For $j \neq t q, 0<j<q^{2}$ and $0<t<q$. we view $(0, j)$ vertex. $(0, j)$ vertex adjacent only $(i, 0)$. The number of $(i, 0)$ vertex is $p^{2}-1$, where $0<i<p^{2}$.

(iv) For $0<t<q$, we view $(0, t q)$ vertex. $(0, t q)$ vertex adjacent $(i, 0),(0, t q),(i, t q)$ where $0<i<p^{2}, 0<t<q$. The number of these vertices is $\left(p^{2}-1\right)+\left(p^{2}-1\right) \cdot(q-1)+(q-2)$, which equals $q p^{2}-2$.

(v) For $j \neq t q, 0<t<q$ and $0<k<p$. we view $(k p, j)$ vertex. $(k p, j)$ vertex adjacent only $(k p, 0)$ vertex. The number of $(k p, 0)$ vertex is $p-1$.

(vi) For $i \neq k p, 0<i<p$ and $0<t<q$, we view $(i, t q)$ vertex. $(i, t q)$ vertex adjacent only $(0, t q)$ vertex. The number of $(0, t q)$ vertex is $q-1$.

(vii) For $0<k<p$ and $0<t<q$, we view $(k p, t q)$ vertex. $(k p, t q)$ vertex adjacent $(0, t q),(k p, 0),(k p, t q)$. The number of these vertices is $(q-1)+(p-1)+(q-1) \cdot(p-1)-1$, which equals $p q-2$.

Among all degrees, the minimum degree $\delta(\Gamma)$ and the maximum degree $\Delta(\Gamma)$ of $\Gamma$ is the number of the smallest and the largest degrees in $\Gamma$. By considering maximum or minimum degrees. We obtain the following result.

Theorem 6. For $p \leq q$, the maximum and the minimum degrees of $\Gamma\left(\mathbb{Z}_{p^{2}} \times \mathbb{Z}_{q^{2}}\right)$ are determined by $\Delta\left(\Gamma\left(\mathbb{Z}_{p^{2}} \times \mathbb{Z}_{q^{2}}\right)\right)=$ $p q^{2}-2$ and $\delta\left(\Gamma\left(\mathbb{Z}_{p^{2}} \times \mathbb{Z}_{q^{2}}\right)\right)=p-1$, respectively. 
Proof. Let $p \leq q$. We consider all vertex of $\Gamma\left(\mathbb{Z}_{p^{2}} \times \mathbb{Z}_{q^{2}}\right)$. By the theorem 5 , let us consider the vertex $(k p, j)$ of $\Gamma\left(\mathbb{Z}_{p^{2}} \times\right.$ $\left.\mathbb{Z}_{q^{2}}\right)$ for any $0<j<q^{2}, j \neq t q$. The vertices adjacent only $(k p, 0)$ for $0<k<p$. The number of $(k p, 0)$ vertex is $p-1$. Thus we have $\delta\left(\Gamma\left(\mathbb{Z}_{p^{2}} \times \mathbb{Z}_{q^{2}}\right)\right)=p-1$. By the theorem 5, let us consider the vertex $(k p, 0)$ of $\Gamma\left(\mathbb{Z}_{p^{2}} \times \mathbb{Z}_{q^{2}}\right)$ for any $0<k<p$. Since $\left(k_{1} p, 0\right) \sim\left(k_{2} p, j\right)$, we have to find the number of $\left(k_{2} p, j\right)$ for $0 \leq j<q^{2}$ and $0<k_{1}, k_{2}<p$. The number of $\left(k_{2} p, j\right)$ vertex is $\left(q^{2}-1\right)+\left(q^{2}-1\right) \cdot(p-1)+(p-2)$, which equals $p q^{2}-2$. Therefore, we have $\Delta\left(\Gamma\left(\mathbb{Z}_{p^{2}} \times \mathbb{Z}_{q^{2}}\right)\right)=p q^{2}-2$.

The degree sequence, denote by $D S(\Gamma)$, is a sequence of degrees of vertices of a graph $\Gamma$. In [12], a new parameter for graphs, namely the irregularity index of $\Gamma$, has been recently defined and denoted by $M W B(\Gamma)$. In fact $M W B(\Gamma)$ is the number of distinct terms in the set $D S(\Gamma)$. At this point, we should note that although this new index is denoted by $t(\Gamma)$ in the paper [12]. Thus, we obtain the following result

Theorem 7. Let $p<q$ be a ring $\mathbb{Z}_{p^{2}} \times \mathbb{Z}_{q^{2}}$. Then the degree sequence and irrequality index of $\Gamma\left(\mathbb{Z}_{p^{2}} \times \mathbb{Z}_{q^{2}}\right)$ are given by

$$
\operatorname{DS}\left(\Gamma\left(\mathbb{Z}_{p^{2}} \times \mathbb{Z}_{q^{2}}\right)\right)=\{\underbrace{p-1}_{q(p-1)(q-1) \text { times }}, \underbrace{q-1}_{q(p-1)(q-1) \text { times }}, \underbrace{p^{2}-1}_{\left(q^{2}-q\right) \text { times }}, \underbrace{p q-2}_{(p-1)(q-1) \text { times }}, \underbrace{q^{2}-1}_{\left(p^{2}-p\right) \text { times }}, \underbrace{q p^{2}-2}_{(q-1) \text { times }}, \underbrace{p q^{2}-2}_{(p-1) \text { times }}\}
$$

and $t\left(\Gamma\left(\mathbb{Z}_{p^{2}} \times \mathbb{Z}_{q^{2}}\right)\right)=4$ where $p=q, t\left(\Gamma\left(\mathbb{Z}_{p^{2}} \times \mathbb{Z}_{q^{2}}\right)\right)=7$ where $p \neq q$.

Proof. The vertices of $\Gamma\left(\mathbb{Z}_{p^{2}} \times \mathbb{Z}_{q^{2}}\right)$ are $(i, 0),(k p, 0),(0, j),(0, t q),(k p, j),(i, t q),(k p, t q)$. By the theorem 5, the degree of vertices are $q^{2}-1, p q^{2}-2, p^{2}-1, q p^{2}-2, p-1, q-1, p q-2$, respectively. Hence, by definition of degree sequence, we clearly obtain the set $D S\left(\Gamma\left(\mathbb{Z}_{p^{2}} \times \mathbb{Z}_{q^{2}}\right)\right)$ as depicted in the theorem.

For any graph $\Gamma$, a subset $D$ of the vertex set $V(\Gamma)$ of a graph $\Gamma$ is called a dominating set if every vertex $V(\Gamma) \backslash D$ is joined to at least one vertex of $D$ by an edge. Additionally, the domination number, denoted by $\gamma(\Gamma)$, is the number of vertices in the smallest dominating set for $\Gamma$ (see [4]). Our next result is about this parameter.

Theorem 8. $\gamma\left(\Gamma\left(\mathbb{Z}_{p^{2}} \times \mathbb{Z}_{q^{2}}\right)\right)=2$.

Proof. If we take any $(k p, 0)$ and $(0, t q)$ vertex, these vertices dominate all the vertices, where $0<k<p, 0<t<q$ and $(k p, 0) \sim(0, t q)$. Therefore we can get $D=\{(k p, 0),(0, t q)\}$ dominating set as $D \subseteq V(G)$. Thus we can see that $\gamma\left(\Gamma\left(\mathbb{Z}_{p^{2}} \times \mathbb{Z}_{q^{2}}\right)\right)=2$.

The coloring of $\Gamma$ is to be an assignment of colors (elements of some set) to the vertices of $\Gamma$, one color to each vertex, so that adjacent vertices are assigned distinct colors. If $n$ colors are used, then the coloring is referred to as $n-$ coloring. If there exists an n-coloring of $\Gamma$, then $\Gamma$ is called $n$-colorable. The minimum number $n$ for which $\Gamma$ is $n$-colorable is called the chromatic number of $\Gamma$ and is denoted by $\chi(\Gamma)$. Moreover, there exists another graph parameter, namely the clique of a graph $\Gamma$. In fact, depending on the vertices, each of the maximal complete subgraphs of $\Gamma$ is called a clique. Additionally, the largest number of vertices in any clique of $\Gamma$ is called the clique number and denoted by $\omega(\Gamma)$. Generally, it is well known that $\chi(\Gamma) \geq \omega(\Gamma)$ for any graph $\Gamma$ (see, for instance [4]). Thus we have the following two theorems.

Theorem 9. The chromatic number of $\Gamma\left(\mathbb{Z}_{p^{2}} \times \mathbb{Z}_{q^{2}}\right)$ is equal to

(i) If $p<q$, then $p q^{2}-1$.

(ii) If $p>q$, then $q p^{2}-1$.

(iii) If $p=q$, then $p^{3}-1$.

Proof. To find the chromatic number, consider the adjacent of $\Gamma\left(\mathbb{Z}_{p^{2}} \times \mathbb{Z}_{q^{2}}\right)$ graph.

(i) For $p<q$, now if we first take account of the vertex $(k p, 0)$ where $0<k<p$, then we have to find the number of adjacent vertex. In other words, we have to find $\operatorname{deg}(k p, 0)$. By the theorem $5, \operatorname{deg}(k p, 0)=p q^{2}-2$. That means the color used for $(k p, 0)$ cannot be used for the remaining vertices. So, let us suppose that the color for $(k p, 0)$ is 
labeled $C$. If we coloring the adjacent vertex, we need to $p q^{2}-1$ color. Other remaining vertex are not adjacent to each other and only adjacent to $(0, t q)$ where $0<t<q$. The number of them are $q p(p-1)$. If $p<q$, then $q p(p-1)<p q^{2}-1$. So that, a total $p q^{2}-1$ colors should be needed, which gives the required cromatic number in the theorem.

(ii) For $p>q$, cromatic number is $q p^{2}-1$ because there is $p q(q-1)<q p^{2}-1$ in a similar way.

(iii) For $p=q$, choromatic number is $p^{3}-1$ when there is $p p(p-1)<p^{2} p-1$ likewise.

Theorem 10. The clique number of $\Gamma\left(\mathbb{Z}_{p^{2}} \times \mathbb{Z}_{q^{2}}\right)$ is $p q-1$.

Proof. Now, let us consider the complete subgraph $A \subseteq \Gamma\left(\mathbb{Z}_{p^{2}} \times \mathbb{Z}_{q^{2}}\right)$. For all distinct vertices $\left(i_{1}, j_{1}\right),\left(i_{2}, j_{2}\right) \in V(A)$, we have

$$
\left(i_{1}, j_{1}\right) .\left(i_{2}, j_{2}\right)=(0,0) \text { i.e. },\left(i_{1}, j_{1}\right) .\left(i_{2}, j_{2}\right) \in E\left(\Gamma\left(\mathbb{Z}_{p^{2}} \times \mathbb{Z}_{q^{2}}\right)\right) \text { for all } i_{1}, i_{2}, j_{1}, j_{2} \text {. }
$$

All of the $(k p, t q)$ vertex adjacent with one another in the graph where $0 \leq k<p, 0 \leq t<q$. The number of this vertex $(q-1)(p-1)+(q-1)+(p-1) . A \subseteq \Gamma\left(\mathbb{Z}_{p^{2}} \times \mathbb{Z}_{q^{2}}\right)$ is a complete subgraph with the vertex set $V(A) . V(A)$ consist of $(k p, t q)$ vertex. It is easy to see that the number of elements in $V(A)$. This is $(q-1)(p-1)+(q-1)+(p-1)$, which equals $p q-1$. So, $\omega\left(\Gamma\left(\mathbb{Z}_{p^{2}} \times \mathbb{Z}_{q^{2}}\right)\right)=p q-1$.

Example 1. By considering the zero-divisor graph $\Gamma\left(\mathbb{Z}_{4} \times \mathbb{Z}_{9}\right)$, the following facts can be see directly as examples of our results.

(i) $\operatorname{diam}\left(\Gamma\left(\mathbb{Z}_{4} \times \mathbb{Z}_{9}\right)\right)=3$.

(ii) $\operatorname{girth}\left(\Gamma\left(\mathbb{Z}_{4} \times \mathbb{Z}_{9}\right)\right)=3$.

(iii) $\operatorname{rad}\left(\Gamma\left(\mathbb{Z}_{4} \times \mathbb{Z}_{9}\right)\right)=2$.

(iv) $\Delta\left(\Gamma\left(\mathbb{Z}_{4} \times \mathbb{Z}_{9}\right)\right)=16$ and $\delta\left(\Gamma\left(\mathbb{Z}_{4} \times \mathbb{Z}_{9}\right)\right)=1$.

(v) $\operatorname{DS}\left(\Gamma\left(\mathbb{Z}_{4} \times \mathbb{Z}_{9}\right)\right)=\{1,1,1,1,1,1,2,2,2,2,3,3,3,3,3,3,4,4,8,8,10,10,16\}$ and $t\left(\Gamma\left(\mathbb{Z}_{4} \times \mathbb{Z}_{9}\right)\right)=7$.

(vi) $\gamma\left(\Gamma\left(\mathbb{Z}_{4} \times \mathbb{Z}_{9}\right)\right)=2$.

(vii) $\chi\left(\Gamma\left(\mathbb{Z}_{4} \times \mathbb{Z}_{9}\right)\right)=17$.

(viii) $\omega\left(\Gamma\left(\mathbb{Z}_{4} \times \mathbb{Z}_{9}\right)\right)=5$.

\section{Competing interests}

The authors declare that they have no competing interests.

\section{Authors' contributions}

All authors have contributed to all parts of the article. All authors read and approved the final manuscript.

\section{References}

[1] D. D. Anderson, M. Naseer, Beck's coloring of a commutative ring, Journal of Algebra 159 (1991), 500-514.

[2] D.F. Anderson, P.S. Livingston, The Zero-divisor Graph of Commutative Ring, Journal of Algebra 217(1999), 434-447.

[3] D.F. Anderson, A. Badawi, On the Zero-Divisor Graph of a Ring, Communications in Algebra 36 (8) (2008), 3073-3092.

[4] Gross, JL, Yellen, J: Handbook of Graph Theory Chapman, Hall/CRC London (2004).

[5] I. Beck, Coloring of Commutating Ring, Journal of Algebra 116(1988), 208-226.

[6] K Ch Das, N.Akgunes and A.S.Cevik, On a graph of monogenic semigroups, Journal of Inequalities and Applications 442013 
[7] K.Patra, Priyanka Pratim Baruah, On the Adjacency Matrix and Neighborhood Associated with Zero-divisor Graph for Direct Product of Finite Commutative Rings International Journal of Computer Applications Technology and Research Volume 2 Issue 3 (2013) 315-323.

[8] N. Akgunes, M. Togan, Some graph theoretical properties over zero-divisor graphs of special finite commutative rings, Adv.Studies Contemp. Math. 22(2) (2012), 305-315.

[9] N. Akgunes, A.S. Cevik, A new bound of radius with irregularity index, Applied Mathematics and Computation 219 (2013) $5750 ? 5753$.

[10] P. Sharma, A. Sharma, R.K. Vats, Analysis of Adjacency Matrix and Neighborhood Associated with Zero Divisor Graph of Finite Commutative Rings, International Journal of Computer Applications 14(3) (2011), 38-42.

[11] S. Akbari, H. R. Maimani, S. Yassemi, When a Zero-Divisor Graph is Planar or a Complete r-Partite Graph, Journal of Algebra 270 (2003), 169-180.

[12] S. Mukwembi, A note on diameter and the degree sequence of a graph. Applied Mathematics 25, (2012) 175-178. 